\title{
Eclipse, transit and occultation geometry of planetary systems at exo-syzygy
}

\author{
Dimitri Veras ${ }^{1 \star}$, Elmé Breedt ${ }^{1}$ \\ ${ }^{1}$ Department of Physics, University of Warwick, Coventry CV4 7AL, UK
}

13 March 2017

\begin{abstract}
Although conjunctions and oppositions frequently occur in planetary systems, eclipserelated phenomena are usually described from an Earth-centric perspective. Space missions to different parts of the Solar system, as well as the mounting number of known exo-planets in habitable zones and the possibility of sending featherweight robot spacecraft to them, prompt broader considerations. Here, we derive the geometry of eclipses, transits and occultations from a primarily exo-Earth viewpoint, and apply the formulation to the Solar system and three types of three-body extrasolar planetary systems: with 1 star and 2 planets (Case I), with 2 stars and 1 planet (Case II), and with 1 planet, 1 star and 1 moon (Case III). We derive the general conditions for total, partial and annular eclipses to occur at exo-syzygy, and implement them in each case in concert with stability criteria. We then apply the formalism to the TRAPPIST-1, Kepler-444 and Kepler-77 systems - the first of which contains multiple potentially habitable planets - and provide reference tables of both Solar system and TRAPPIST1 syzygy properties. We conclude by detailing a basic algebraic algorithm which can be used to quickly characterize eclipse properties in any three-body system.
\end{abstract}

Key words: eclipses - occultations - celestial mechanics - methods: analytical planets and satellites: general - planet and satellites: dynamical evolution and stability

\section{INTRODUCTION}

By the time Nicolaus Copernicus postulated that the Earth revolves around the Sun - and not vice versa - humans had already been studying eclipses for thousands of years (Li \& Zhang 1998). Indeed, one of the oldest-known astrophysical phenomena is syzygy, when three or more celestial bodies become co-linear. Although often defined in terms of the Sun, Moon and Earth only, syzygys could involve other Solar system bodies, and more than three total. McDonald (1986) computed the frequencies of a variety of Solar system syzygys, and Pease \& Glenn (2016) provided specific examples, such as when the Sun, Venus, Earth, Jupiter and Saturn achieved syzygy in the year 1683, and when the Sun, Venus, Jupiter and Saturn will achieve syzygy in the year 2040 .

In the argot of astronomers, the terms eclipse, transit, and occultation have come to represent syzygy in different contexts (e.g. the Sun is eclipsed, whereas exo-planets transit, and stars are occulted). Semantics aside, the geometrical configuration provided by a syzygy yields invaluable information. The Sun's corona is visible from Earth only during particular types of syzygys, the rings around the as-

* E-mail: d.veras@warwick.ac.uk teroid Chariklo were detected only because of a different type of syzygy (Braga-Ribas et al. 2014), and the first putative detection of rings around an exo-moon were made possible because of syzygys (Kenworthy \& Mamaiek 2015). In fact, exo-planetary systems containing multiple planets which transit their parent star or stars have the potential to be "most information-rich planetary systems besides our own solar system" (Ragozzine \& Holman 2010). The idea of exo-syzygy is not just a possibility, but a reality: exo-planets like Kepler-1647 b have already achieved syzygy with both of its parent stars and the Earth (Kostov et al. 2016).

Almost ubiquitously, syzygys have been studied by assuming a viewpoint on or close to the Earth's surface. However, the motivation for studying syzygys in a more general context has now received added impetus with (i) the numerous space missions that visit other parts of the Solar system (and beam back data from a variety of viewpoints), and (ii) the febrile desire to find habitable planets and understand what life is like on them. Cassini, MESSENGER and New Horizons are but a few of the robotic spacecraft which have provided us with different perspectives. The great fortune that our nearest stellar neighbour (Proxima Centuri) happens to host a detectable planet in the habitable zone (Anglada-Escudé et al. 2016) has provided an ideal flyby 
candidate for the Breakthrough Starshot mission! 1 , which has already received significant financial backing.

Here, we formulate a geometry for three-body syzygys in a general-enough context for wide applications and which yields an easy-to-apply collection of formulae given only the radii and mutual distances of the three bodies. We then apply the formulae to a wide variety of Solar system syzygys and extrasolar syzygys. We begin in Section 2 by establishing our setup. We then place eclipses into context for three different combinations of stars, planets and moons in Sections 3-5 by providing limits in each case from the results of the derivations in Appendices A and B and from stability criteria. We apply our formulae to a plethora of specific cases for the Solar system in Section 6 and for extrasolar systems in Sections 7-8. We conclude with a useful algorithm, including a user-friendly flow chart, in Section 9.

All data used in the applications are taken from two sources unless otherwise specified: the Jet Propulsion Laboratory Solar System Dynamics websit $2^{2}$ and the Exoplanet Data Explore1 3 .

\section{SETUP}

We model planetary systems that include three spherical bodies, at least one of which is a star. Because we consider the systems only at syzygy, no motion is assumed, allowing for a general treatment with wide applicability.

\section{$2.1 \quad$ Nomenclature}

We denote the star as the "primary", the body on or around which an observer/detector might see an eclipse as the "target", and the occulting/transiting/eclipsing body as the "occulter". The occulter may be a planet (Case I), star (Case II) or moon (Case III), and we consider these individual cases in turn in Sections 3-5. We denote the radii of the primary, occulter and target to be, respectively $R_{1}, R_{2}$ and $R_{3}$, and the pairwise distances between the centres of the objects as $r_{12}, r_{23}$ and $r_{13}$. We assume that the primary is the largest of the three objects, such that $R_{1}>R_{2}$ and $R_{1}>R_{3}$, but make no assumptions about the relative sizes of the occulter and target.

All results are derived in terms of the radii and mutual distances only. The masses of the bodies $M_{1}, M_{2}$ and $M_{3}$ rarely factor in the equations, but regardless are convenient identifiers for diagrams and will primarily be used for that purpose.

\section{$2.2 \quad$ Mutual distances}

The values of any two of $r_{12}, r_{13}$ and $r_{23}$ may be given, allowing for trivial computation of the third value. For Cases I and II, when the occulter is a planet or star, the astrocentric distances $r_{12}$ and $r_{13}$ are often known, whereas for Case III, when the occulter is a moon, $r_{13}$ and $r_{23}$ are often known from observations.

\footnotetext{
1 http://breakthroughinitiatives.org/

2 http://ssd.jpl.nasa.gov/

3 http://exoplanets.org/
}

However, in both observational and theoretical studies, orbits with respect to centres of mass (as in barycentric and Jacobi coordinates) are sometimes more valuable. Denote $r_{123}$ as the distance of the target to the centre of mass of the primary and occulter. Then

$r_{13}=r_{123}+\left(\frac{M_{1}}{M_{1}+M_{2}}\right) r_{12}$.

Observations from Earth rarely catch three-bodies in an exo-planetary system in syzygy. Therefore, instantaneously measured or estimated mutual distances may not be as helpful as orbital parameters, such as semimajor axis $a$, eccentricity $e$ and true anomaly $\Pi$. They are related to separation as

$r_{12}=\frac{a_{12}\left(1-e_{12}^{2}\right)}{1+e_{12} \cos \left[\Pi_{12}\left(t_{\mathrm{s}}\right)\right]}$

with similar forms for $r_{23}, r_{13}$ and $r_{123}$.

The values of $a$ and $e$ can be treated as fixed on orbital timescales, whereas $\Pi$ is a proxy for time evolution. In threebody systems, at some points along the mutual orbits - at nodal intersections - the three bodies achieve syzygy. The inclinations, longitudes of ascending nodes and arguments of pericentre of the bodies help determine eclipse details associated with motion (such as duration length); here we consider only the static case at syzygy, and hence are unconcerned with these other parameters, as well as the time at which the true anomalies achieve syzygy.

\section{$2.3 \quad$ Radiation cones}

In all cases, the radiation emanating from the primary will form two different types of cones with the occulter, because the latter is smaller than the former. The first type of cone, yielding total and annular eclipses, is formed from outer or external tangent lines (Fig. 1] for derivations, see Appendix A). The second type of cone, yielding partial eclipses, form from the inner or internal tangent lines (Fig. 2 for derivations, see Appendix B).

\section{CASE I: PLANET OCCULTER}

We now apply our geometrical formalism to three general cases, starting with that of a planet occulter. One specific example of this case would be Mercury transiting the Sun as viewed from Earth. In what follows, we note that given semimajor axis $a$ and eccentricity $e$, one can generate bounds on the mutual distances by considering their pericentric $[a(1-e)]$ and apocentric $[a(1+e)]$ values.

\subsection{Never total eclipses}

From equation (A7), a total eclipse can never occur if

$\max (h+n)<\min \left(r_{13}\right)-R_{3}$

or

$\frac{R_{1}}{R_{1}-R_{2}}<\left(\frac{a_{13}}{a_{12}}\right)\left(\frac{1-e_{13}-\frac{R_{3}}{a_{13}}}{1+e_{12}}\right)$. 


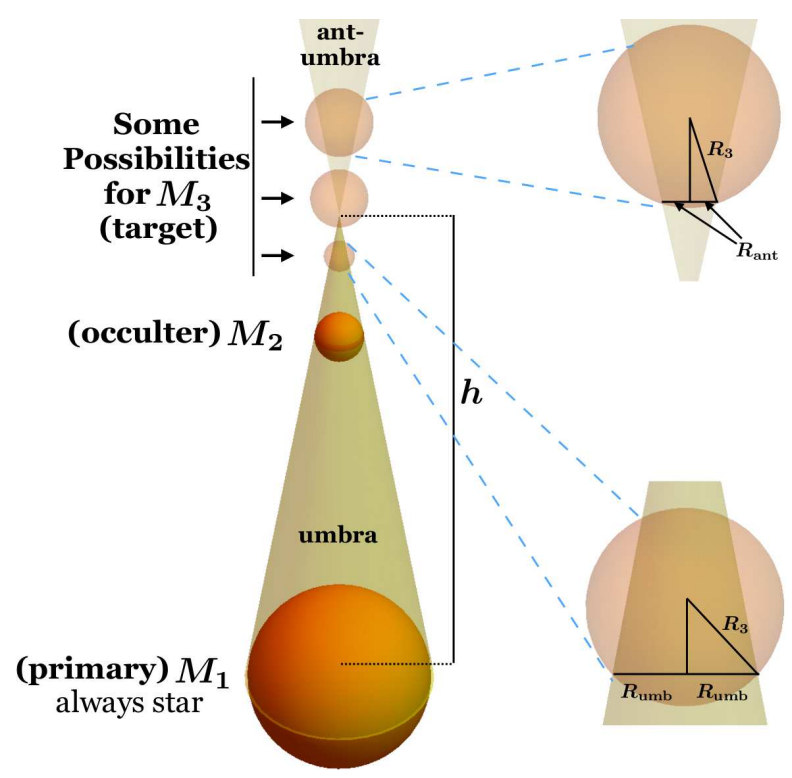

Figure 1. A geometrical sketch for the umbra and antumbra. The cone defined by the radiation emitted from the spherical primary has a height $h$ and circumscribes the occulter. If the target intersects the umbral cone at syzygy, then a total eclipse will occur as seen by an observer on the target. The projected area of the total eclipse area is defined by the radius $R_{\text {umb }}$. Otherwise, an annular eclipse will occur, with a projected radius of $R_{\text {ant }}$. The primary is always larger than the occulter and target.

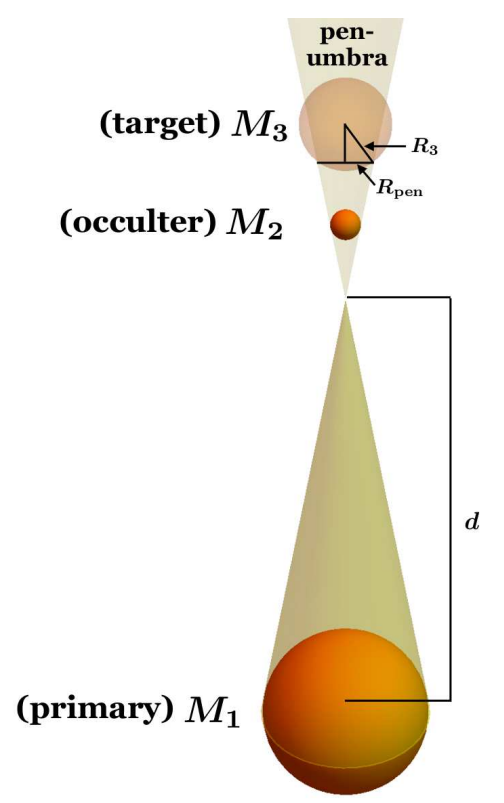

Figure 2. A geometrical sketch for the penumbral shadow, which, in contrast to Fig. 1 is bounded by internal tangent lines between the primary and occulter (the external tangent lines of Fig. [1 produce a longer cone). The height of the cone here is $d$. The illustration depicts the shadow falling on only part of the target; for a distant-enough or small-enough target, $R_{\text {pen }}$ would no longer be defined.
In all cases, because the primary is a star and the target must lie at a sufficiently large distance from the occulter to remain stable, the term $R_{3} / a_{13}$ is negligible. For example, in an extreme case, for a Jupiter-sized planet at 0.05 au away from a star (with another planet in-between), then $R_{3} / a_{13} \approx 1 \%$.

The ratio on the left-hand side of the equation could take on a variety of values depending on the nature of the star. If the star is a giant branch star, then $R_{1} \gg R_{2}$ and

$\left[1+\frac{R_{2}}{R_{1}}\right] a_{12}\left(1+e_{12}\right) \lesssim a_{13}\left(1-e_{13}\right)$

which is effectively a condition on crossing orbits and hence is always true for our setup. Therefore, total eclipses cannot occur in extrasolar systems with two planets and one giant star.

If instead the star is a main sequence star larger than a red dwarf, then it is useful to consider the fact that the planet's size is bounded according to $\max \left(R_{2}\right) \approx 0.01 R_{\odot}$. For a red dwarf, around which potentially habitable planets like Proxima b have been discovered Anglada-Escudé et al. $2016), \max \left(R_{2}\right) \approx 0.1 R_{\odot}$. In either approximation, for any main sequence stars, equation (5) holds and total eclipses can never occur.

\subsection{Always total eclipses}

For smaller stars, like white dwarfs, we now consider the opposite extreme.

A total eclipse will always occur if

$\min (h+n) \geqslant \max \left(r_{13}\right)-R_{3}$

or

$\frac{R_{1}}{R_{1}-R_{2}} \geqslant\left(\frac{a_{13}}{a_{12}}\right)\left(\frac{1+e_{13}-\frac{R_{3}}{a_{13}}}{1-e_{12}}\right)$,

which we approximate as

$a_{12}\left(1-e_{12}\right) \gtrsim a_{13}\left(1+e_{13}\right)\left[1-\frac{R_{2}}{R_{1}}\right]$.

by removing the $R_{3} / a_{13}$ term but otherwise not making any assumptions about the relative values of $R_{1}$ and $R_{2}$. Equation (8) holds true when the term in the square brackets is sufficiently small.

Therefore, if the primary and occulter are about the same size (such as an Earth-like planet orbiting a white dwarf), then a total eclipse always occurs. Otherwise, the bracketed term determines the factor by which the apocentre of the outer planet must be virtually reduced in order to satisfy the equation. Usefully, the equation explicitly contains the pericentre of the inner planet and the apocentre of the outer planet.

\section{CASE II: STELLAR OCCULTER}

Because circumbinary planets are now known to be common and will continue to represent a source for some of the most fascinating planetary systems in the foreseeable future (Armstrong et al. 2014; Martin \& Triaud 2015; Sahlmann et al. 2015), the case of a stellar occulter is important to consider. When a system contains two stars and 


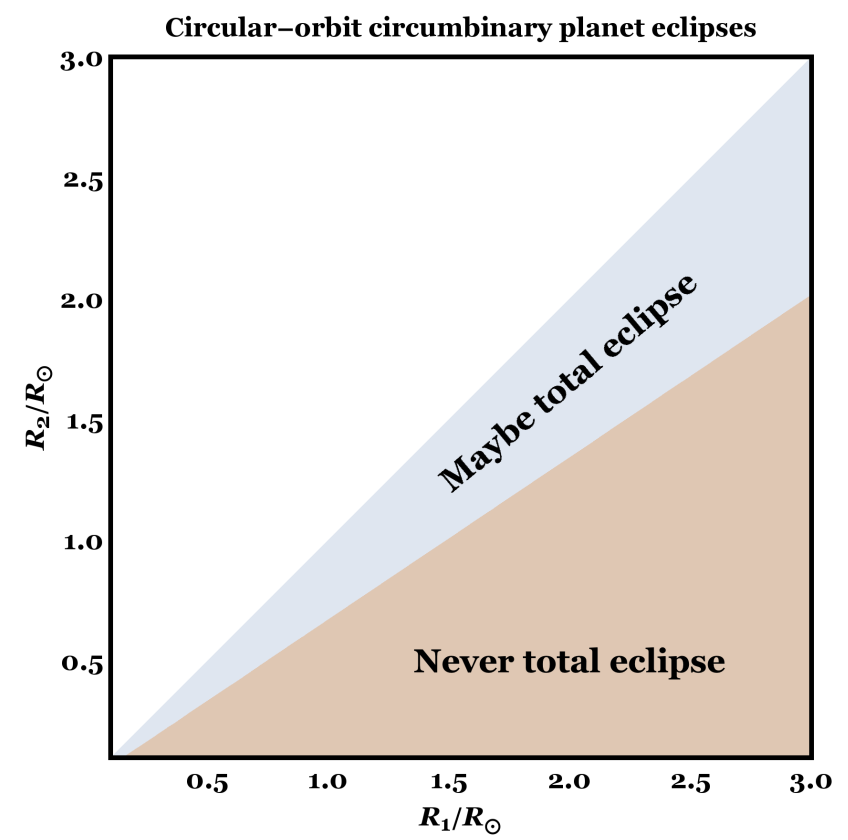

Figure 3. Where total eclipses in circumbinary systems cannot occur. Here circular orbits $\left(e_{12}=e_{123}=0\right)$ and equal stellar densities are assumed. The units of $R_{1}$ and $R_{2}$ were arbitrarily chosen, and in fact may be any other unit. This plot was derived from equation (13).

one circumbinary planet, then the planet's orbital elements are usually measured with respect to the centre of mass of the stars (and contain a subscript of " 123 ", as indicated in Section 2).

\subsection{Never total eclipses}

Hence, combining the condition for total eclipses to never occur (equation 3) with equation (1) yields

$\min (h+n)<\max \left(r_{123}\right)+\max \left(\frac{r_{12} M_{1}}{M_{1}+M_{2}}\right)-R_{3}$.

This expression, under the reasonable assumption $e_{12}=0$ for circumbinary planet host stars, gives

$\frac{R_{1}}{R_{1}-R_{2}}<\frac{a_{123}\left(1-e_{123}\right)}{a_{12}}+\frac{M_{1}}{M_{1}+M_{2}}-\frac{R_{3}}{a_{12}}$.

The last term can be neglected because $R_{3} \ll R_{1}<a_{12}$. We can go further and consider cases in which both stars have the same density. Then equation (10) becomes

$\frac{R_{1} R_{2}\left(R_{1}^{2}+R_{2}^{2}\right)}{\left(R_{1}-R_{2}\right)\left(R_{1}^{3}+R_{2}^{3}\right)}<\frac{a_{123}\left(1-e_{123}\right)}{a_{12}}$,

which illustrates that to ensure a total eclipse will never occur, a planet's pericentre must be sufficiently distant, and the stellar orbit must be sufficiently tight.

We can now take the relation even further. If we know that the planet is on a circular orbit $\left(e_{123}=0\right)$, then we may use the stability boundary of equation 3 of Holman \& Wiegert (1999), which yields (still assuming $\left.e_{12}=0\right)$,

$$
\begin{aligned}
& \min \left(a_{123}\right) \approx \\
& a_{12}\left[1.60+4.12\left(\frac{M_{2}}{M_{1}+M_{2}}\right)-5.09\left(\frac{M_{2}}{M_{1}+M_{2}}\right)^{2}\right] .
\end{aligned}
$$

This boundary, along with the assumptions of circularity and equal stellar densities, allow us to write the condition for ensuring total eclipses on the target in terms of only the stellar radii as

$$
\frac{R_{1}^{6} R_{2}-3.12 R_{1}^{4} R_{2}^{3}+5.12 R_{1}^{3} R_{2}^{4}+1.97 R_{1} R_{2}^{6}-0.97 R_{2}^{7}}{\left(R_{1}-R_{2}\right)\left(R_{1}^{3}+R_{2}^{3}\right)^{2}}<1.6 .
$$

We plot Fig. 3 from equation (13), and recall that our setup requires $R_{1}>R_{2}$. The plot emphasizes that the radii of both stars must be close to each other in order to maintain the possibility of a total eclipse.

\subsection{Always total eclipses}

In order to guarantee a total eclipse, we combine equation (6) with equation (11) to yield

$\frac{R_{1}}{R_{1}-R_{2}} \geqslant \frac{a_{123}\left(1+e_{123}\right)}{a_{12}}+\frac{M_{1}}{M_{1}+M_{2}}-\frac{R_{3}}{a_{12}}$.

This equation illustrates that when $R_{1}=R_{2}$, a total eclipse will always occur. We can again neglect the last term. Under the assumption of equivalent stellar densities, we find

$\frac{R_{1} R_{2}\left(R_{1}^{2}+R_{2}^{2}\right)}{\left(R_{1}-R_{2}\right)\left(R_{1}^{3}+R_{2}^{3}\right)} \geqslant \frac{a_{123}\left(1+e_{123}\right)}{a_{12}}$.

\section{CASE III: MOON OCCULTER}

For a moon occulter (such as the Sun-Earth-Moon system), we follow a similar procedure as to the last two sections in order to provide relations which establish parameter space regimes in which total eclipses can never or always occur.

First we note that additional constraints can be imposed on the moon's orbital size, from both below and above. These constraints may be used with the formulae below depending on what quantities are known. As detailed in Payne et al. (2016), moons can exist in stable orbits if they reside somewhere outside of the Roche, or disruption, radius of the planet, and inside about one-half of a Hill radius (see also Hamilton \& Krivov 1997, Domingos et al. 2006 and Donnison 2010). Hence,

$k_{\rho}\left(\frac{M_{1}}{\rho_{2}}\right)^{1 / 3}<r_{23}<k_{\mathrm{H}} a_{13}\left(1-e_{13}\right)\left(\frac{M_{3}}{3 M_{1}}\right)^{1 / 3}$

where $\rho$ refers to density, and $k_{\rho}$ and $k_{\mathrm{H}}$ are constants. $k_{\rho}$ is a constant which can take on a variety of values depending on the body's shape, composition and spin, and typically span the range 0.78-1.53 (Table 1 of Veras et al. 2017). Although a common value of $k_{\mathrm{H}}$ is 0.5 , it is dependent on the direction of revolution and other properties of the moon.

\subsection{Never total eclipses}

In the context of typically-known orbital elements in systems with a moon, equation (3) becomes 
$\left(\frac{R_{1}}{R_{1}-R_{2}}\right) \max \left(r_{13}-r_{23}\right)<\min \left(r_{13}\right)-R_{3}$,

which can be simplified, by neglecting a $R_{3} / a_{13}$ term, to

$\frac{a_{23}}{a_{13}}>\frac{1+e_{13}-\left(1-e_{13}\right)\left[\frac{R_{1}-R_{2}}{R_{1}}\right]}{1-e_{23}}$.

If both orbits are circular, then equation 21 reduces to the compact form

$\frac{a_{23}}{a_{13}}>\frac{R_{2}}{R_{1}}$.

For the case of the Sun, Moon and Earth, both sides of equation (19) are nearly equal (to within a few per cent of about 0.0025). Hence, because the equation sometimes holds, the Moon sometimes produces total eclipses, and sometimes does not.

\subsection{Always total eclipses}

In a similar fashion, the condition to ensure that total eclipses will occur (equation 6) gives

$\left(\frac{R_{1}}{R_{1}-R_{2}}\right) \min \left(r_{13}-r_{23}\right) \geqslant \max \left(r_{13}\right)-R_{3}$,

or, by neglecting a $R_{3} / a_{13}$ term,

$\frac{a_{23}}{a_{13}} \leqslant \frac{1-e_{13}-\left(1+e_{13}\right)\left[\frac{R_{1}-R_{2}}{R_{1}}\right]}{1+e_{23}}$.

\section{APPLICATION TO SOLAR SYSTEM}

We begin applying our general formalism to real systems by considering three-body subsets of the Solar system. Table 6 lists some properties of these subsystems. For almost every subsystem, we provide two extreme cases, when the distance between the occulter and target are minimized and maximized. We achieve these limits by computing the relevant ratios of orbital pericentres and apocentres.

We emphasize that the values in the table are theoretical estimates based on our formalism here and do not take into account the many complications that exist in reality, such as oblateness and albedo effects. Nevertheless, the table reveals interesting facets about eclipses.

\subsection{Observers on Earth}

The first ten cases are applicable to observers on Earth. The disc of Venus appears 5-10 times larger than the disc of Mercury when transiting the Sun. Mercury's angular diameter can change significantly - by about a quarter - depending on its distance from both the Sun and the Earth. Both Mercury and Venus generate transit depths which are less than $10^{-3}$, and antumbral cones which completely engulf the Earth.

The Sun-Earth-Moon cases emphasize how unusual the situation is in which humans find themselves. Depending on the Sun-Earth and Earth-Moon distances, either a total eclipse or annular eclipse can occul The minimum eclipse depth is about 0.85 , which demonstrates that at a minimum,

${ }^{4}$ In rare cases, a "hybrid" or "mixed" eclipse occurs when both

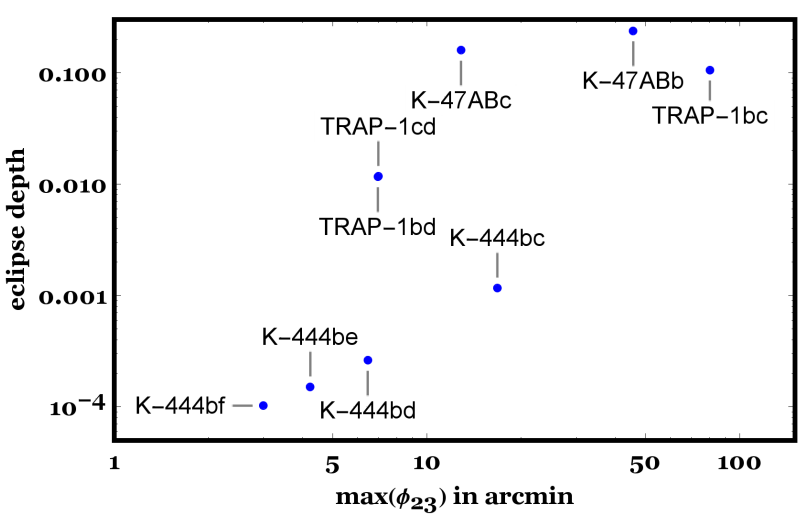

Figure 4. The eclipse depths and angular diameters of some exoplanetary syzygys in the TRAPPIST-1, Kepler-444 and Kepler-47 systems. Kepler- 47 is a circumbinary system and the other two are single-star systems. The letters indicate which bodies are in syzygy.

92 per cent of the Sun's disc is covered by the Moon. Neither the umbral nor antumbral cones ever engulf the Earth. In fact, the projected radius of the shadow cast on Earth does not exceed $150 \mathrm{~km}$ (or 93 miles) 5 . The penumbral cone also does not engulf the Earth, and produces a much larger shadow with a projected radius of 3380-3640 km (2100-2260 miles). Compared to every other table entry with the Sun as primary, the angular diameter of the moon on the Sun's disc is large: about half of a degree. Only observers on Mars watching Phobos transit the Sun would see an angular diameter which is a third of that of the Moon on the Sun. If an astronaut was standing on the Moon during an Earth eclipse of the Sun, they would always see a total eclipse.

\subsection{Other perspectives}

Observatories on Mars must fit themselves into smaller targets - on the order of just $\mathrm{km}$ or tens of $\mathrm{km} \mathrm{-} \mathrm{in} \mathrm{order}$ to see annular eclipses produced by that planet's satellites. Phobos' eclipses are substantial enough to block out about one-third to one-half of the Sun's light. The eclipses due to the Earth as viewed on Mars are less prominent, and are roughly comparable to Venus transits of the Sun as viewed on Earth.

Regarding the outer Solar system, Jupiter is large enough to easily create total eclipses on all of the Galilean satellites. Pluto and Charon create total eclipses on one another, with the umbral shadow of Pluto completely engulfing Charon. If Planet Nine de la Fuente Marcos \& de la Fuente Marcos 2014; Iorio 2014; Batygin \& Brown 2016) exists (assuming a radius of $1.5 R_{\oplus}$ and a separation of $750 \mathrm{au}$ ), then the view from that distant wanderer during a Jupiter eclipse would see a

total and annular eclipses occur during the same event. More technically, the transition into and out of syzygy creates changes in all mutual distances such that during this transition some parts of the Earth satisfy the upper branch of equation (A7) and others satisfy the lower branch of that equation.

5 Actually a more accurate upper limit, due to effects not considered here, is $140 \mathrm{~km}$. 


\begin{tabular}{|c|c|c|c|c|c|c|c|c|c|c|}
\hline primary & occulter & target & $r_{23}$ & $r_{12}$ & $\begin{array}{l}\text { umbra or } \\
\text { antumbra }\end{array}$ & $\begin{array}{c}\text { engulfed } \\
\text { in umb/ant? }\end{array}$ & $\begin{array}{l}\text { engulfed } \\
\text { in pen? }\end{array}$ & $\begin{array}{c}R_{\text {umb }} \text { or } \\
R_{\text {ant }}(\mathrm{km})\end{array}$ & $\max \left(\phi_{23}\right)$ & $\max (g)$ \\
\hline Sun & Mercury & Earth & $\min$ & $\max$ & ant & yes & yes & - & $0.217^{\prime}$ & $4.45 \times 10^{-5}$ \\
\hline Sun & Mercury & Earth & $\max$ & $\min$ & ant & yes & yes & - & $0.158^{\prime}$ & $2.53 \times 10^{-5}$ \\
\hline Sun & Venus & Earth & $\min$ & $\max$ & ant & yes & yes & - & $1.09^{\prime}$ & $1.12 \times 10^{-3}$ \\
\hline Sun & Venus & Earth & $\max$ & $\min$ & ant & yes & yes & - & 0.933 & $8.79 \times 10^{-4}$ \\
\hline Sun & Moon & Earth & $\min$ & $\max$ & ant & no & no & 149 & $29.9^{\prime}$ & 0.847 \\
\hline Sun & Moon & Earth & $\max$ & $\min$ & umb & no & no & 110 & $31.5^{\prime}$ & 1.0 \\
\hline Jupiter & Io & Earth & $\min$ & $\max$ & ant & yes & yes & - & $0.0213^{\prime}$ & $6.80 \times 10^{-4}$ \\
\hline Jupiter & Europa & Earth & $\min$ & $\max$ & ant & yes & yes & - & 0.0183 & $5.00 \times 10^{-4}$ \\
\hline Jupiter & Ganymede & Earth & $\min$ & $\max$ & ant & yes & yes & - & $0.0308^{\prime}$ & $1.42 \times 10^{-3}$ \\
\hline Jupiter & Callisto & Earth & $\min$ & $\max$ & ant & yes & yes & - & 0.0030' & $1.31 \times 10^{-5}$ \\
\hline Sun & Earth & Moon & $\min$ & $\min$ & umb & yes & yes & - & $32.5^{\prime}$ & 1.0 \\
\hline Sun & Earth & Moon & $\max$ & $\max$ & umb & yes & yes & - & $31.4^{\prime}$ & 1.0 \\
\hline Sun & Earth & Mars & $\min$ & $\max$ & ant & yes & yes & - & $0.803^{\prime}$ & $1.20 \times 10^{-3}$ \\
\hline Sun & Earth & Mars & $\max$ & $\min$ & ant & yes & yes & - & $0.429^{\prime}$ & $4.99 \times 10^{-4}$ \\
\hline Sun & Phobos & Mars & $\min$ & $\min$ & ant & no & no & 9.34 & $12.5^{\prime}$ & 0.289 \\
\hline Sun & Phobos & Mars & $\max$ & $\max$ & ant & no & no & 5.02 & $13.1^{\prime}$ & 0.463 \\
\hline Sun & Deimos & Mars & $\min$ & $\min$ & ant & no & no & 61.4 & $2.12^{\prime}$ & $8.40 \times 10^{-3}$ \\
\hline Sun & Deimos & Mars & $\max$ & $\max$ & ant & no & no & 49.8 & 2.12 & $1.22 \times 10^{-2}$ \\
\hline Sun & Earth & Jupiter & $\min$ & $\max$ & ant & yes & yes & - & $0.0744^{\prime}$ & $1.33 \times 10^{-4}$ \\
\hline Sun & Earth & Jupiter & $\max$ & $\min$ & ant & yes & yes & - & 0.0655 & $1.25 \times 10^{-4}$ \\
\hline Sun & Io & Jupiter & $\min$ & $\min$ & umb & no & no & 1490 & $6.46^{\prime}$ & 1.0 \\
\hline Sun & Io & Jupiter & $\max$ & $\max$ & umb & no & no & 1520 & $5.86^{\prime}$ & 1.0 \\
\hline Sun & Europa & Jupiter & $\min$ & $\min$ & umb & no & no & 990 & $6.46^{\prime}$ & 1.0 \\
\hline Sun & Europa & Jupiter & $\max$ & $\max$ & umb & no & no & 1050 & $5.86^{\prime}$ & 1.0 \\
\hline Sun & Ganymede & Jupiter & $\min$ & $\min$ & umb & no & no & 1690 & $6.46^{\prime}$ & 1.0 \\
\hline Sun & Ganymede & Jupiter & $\max$ & $\max$ & umb & no & no & 1780 & $5.86^{\prime}$ & 1.0 \\
\hline Sun & Callisto & Jupiter & $\min$ & $\min$ & umb & no & no & 694 & $6.46^{\prime}$ & 1.0 \\
\hline Sun & Callisto & Jupiter & $\max$ & $\max$ & umb & no & no & 877 & $5.86^{\prime}$ & 1.0 \\
\hline Sun & Pluto & Charon & circ & $\min$ & umb & yes & yes & - & $1.08^{\prime}$ & 1.0 \\
\hline Sun & Pluto & Charon & circ & $\max$ & umb & yes & yes & - & $0.649^{\prime}$ & 1.0 \\
\hline Sun & Charon & Pluto & circ & $\min$ & umb & no & no & 603 & $1.08^{\prime}$ & 1.0 \\
\hline Sun & Charon & Pluto & circ & $\max$ & umb & no & no & 604 & $0.649^{\prime}$ & 1.0 \\
\hline Sun & Jupiter & Pl. Nine & circ & $\min$ & ant & yes & yes & - & 0.0043 & 0.010 \\
\hline Jupiter & Io & Europa & $\min$ & $\max$ & ant & yes & yes & - & 52.3 & $5.18 \times 10^{-3}$ \\
\hline Jupiter & Io & Europa & $\max$ & $\min$ & ant & yes & yes & - & $48.9^{\prime}$ & $4.73 \times 10^{-3}$ \\
\hline Jupiter & Io & Ganymede & $\min$ & $\max$ & ant & yes & yes & - & 19.5 & $1.87 \times 10^{-3}$ \\
\hline Jupiter & Io & Ganymede & $\max$ & $\min$ & ant & yes & yes & - & $19.3^{\prime}$ & $1.84 \times 10^{-3}$ \\
\hline Jupiter & Ganymede & Callisto & $\min$ & $\max$ & ant & yes & yes & - & $22.8^{\prime}$ & $7.81 \times 10^{-3}$ \\
\hline Jupiter & Ganymede & Callisto & $\max$ & $\min$ & ant & yes & yes & - & $21.9^{\prime}$ & $7.46 \times 10^{-3}$ \\
\hline Saturn & Encleadus & Tethys & circ & circ & ant & yes & yes & - & $30.9^{\prime}$ & $5.09 \times 10^{-4}$ \\
\hline Saturn & Titan & Hyperion & $\min$ & $\max$ & ant & yes & yes & - & $84.7^{\prime}$ & $9.62 \times 10^{-2}$ \\
\hline Saturn & Titan & Hyperion & $\max$ & $\min$ & ant & yes & yes & - & $50.7^{\prime}$ & $3.78 \times 10^{-2}$ \\
\hline Uranus & Titania & Oberon & $\min$ & $\max$ & ant & yes & yes & - & $37.4^{\prime}$ & $1.55 \times 10^{-2}$ \\
\hline Uranus & Titania & Oberon & $\max$ & $\min$ & ant & yes & yes & - & $36.7^{\prime}$ & $1.51 \times 10^{-2}$ \\
\hline Neptune & Triton & Nereid & circ & $\max$ & ant & yes & yes & - & 9.12 & $5.49 \times 10^{-3}$ \\
\hline Neptune & Triton & Nereid & circ & $\min$ & ant & yes & yes & - & $1.00^{\prime}$ & $3.25 \times 10^{-3}$ \\
\hline
\end{tabular}

Table 1. Eclipse properties of three-body subsystems within the Solar system at syzygy. A value of "circ" in the fourth and fifth columns indicates a circular orbit. The penultimate column provides the maximum possible angular diameter as observed on the target, and the final column gives the maximum eclipse depth (from equation A30). When this latter value is equal to unity, a total eclipse occurs for an observer co-linear with the syzygy.

transit depth of 1 per cent, despite an angular diameter of just one-quarter of an arcsecond.

The last 13 rows of the table describe situations where Jupiter, Saturn, Uranus or Neptune is considered to be the primary and the occulter and target are two attached moons. In all cases, an annular eclipse occurs, and the antumbral shadow encompasses the target. The transit depths are within an order of $10^{-3}$ and the angular diameters are on the order of tens of arcminutes. The largest value of $\phi_{23}$ (over one degree) occurs with Titan and Hyperion, which are highly disparate in size (Titan is 19 times greater in radius) and yet very close to each other (in fact within a 4:3 mean motion resonance). 


\begin{tabular}{|c|c|c|c|c|c|c|c|c|c|c|}
\hline primary & occulter & target & $r_{23}$ & $r_{12}$ & $\begin{array}{l}\text { umbra or } \\
\text { antumbra }\end{array}$ & $\begin{array}{c}\text { engulfed } \\
\text { in umb/ant? }\end{array}$ & $\begin{array}{l}\text { engulfed } \\
\text { in pen? }\end{array}$ & $\begin{array}{c}R_{\text {umb }} \text { or } \\
R_{\text {ant }}(\mathrm{km})\end{array}$ & $\max \left(\phi_{23}\right)$ & $\max (g)$ \\
\hline TRAPPIST-1 & Planet $\mathrm{b}$ & Planet e & $\operatorname{circ}$ & circ & ant & yes & yes & - & $18.7^{\prime}$ & $1.96 \times 10^{-2}$ \\
\hline TRAPPIST-1 & Planet c & Planet e & circ & circ & ant & yes & yes & - & $23.9^{\prime}$ & $3.18 \times 10^{-2}$ \\
\hline TRAPPIST-1 & Planet d & Planet e & circ & circ & ant & yes & yes & - & 32.5 & $5.89 \times 10^{-2}$ \\
\hline TRAPPIST-1 & Planet b & Planet $\mathrm{f}$ & circ & circ & ant & yes & yes & - & $12.3^{\prime}$ & $1.46 \times 10^{-2}$ \\
\hline TRAPPIST-1 & Planet c & Planet $\mathrm{f}$ & circ & circ & ant & yes & yes & - & $14.1^{\prime}$ & $1.93 \times 10^{-2}$ \\
\hline TRAPPIST- 1 & Planet d & Planet $\mathrm{f}$ & circ & circ & ant & yes & yes & - & $14.2^{\prime}$ & $1.96 \times 10^{-2}$ \\
\hline TRAPPIST- 1 & Planet e & Planet $\mathrm{f}$ & circ & circ & ant & yes & yes & - & 30.0 & $8.78 \times 10^{-2}$ \\
\hline TRAPPIST-1 & Planet $\mathrm{b}$ & Planet $g$ & circ & circ & ant & yes & yes & - & 9.37 & $1.27 \times 10^{-2}$ \\
\hline TRAPPIST-1 & Planet c & Planet g & circ & circ & ant & yes & yes & - & $10.3^{\prime}$ & $1.54 \times 10^{-2}$ \\
\hline TRAPPIST-1 & Planet d & Planet $\mathrm{g}$ & circ & circ & ant & yes & yes & - & $9.44^{\prime}$ & $1.28 \times 10^{-2}$ \\
\hline TRAPPIST-1 & Planet e & Planet $\mathrm{g}$ & circ & circ & ant & yes & yes & - & $15.9^{\prime}$ & $3.63 \times 10^{-2}$ \\
\hline TRAPPIST-1 & Planet $\mathrm{f}$ & Planet $\mathrm{g}$ & circ & circ & ant & yes & yes & - & 38.5 & $2.14 \times 10^{-2}$ \\
\hline
\end{tabular}

Table 2. Eclipse properties of three potentially habitable planets (planets e, $\mathrm{f}$ and $\mathrm{g}$ ) in the TRAPPIST-1 system. The columns are equivalent to those in Table 1 for easy comparison; the penultimate column provides the maximum possible angular diameter as observed on the target, and the final column gives the maximum eclipse depth (from equation A30). All planets are assumed to be coplanar and have circular orbits. Data for these computations is taken from Gillon et al. (2017).

\section{APPLICATION TO KNOWN EXO-SYSTEMS}

Exo-planetary systems are now known to host a wide variety of exo-planets, with masses ranging from 1.6 Lunar masses Wolszczan 1994; Konacki \& Wolszczan 2003) all the way into the stellar regime. About 41 per cent of all known planets are in multiple-planet systems, which lead to eclipses of Type I. A couple dozen planets have two stellar hosts and orbit in a circumbinary fashion, leading to Type II eclipses. Although no exo-moons have yet been confirmed (for Type III eclipses), vigorous searches have been undertaken (Heller 2017), even despite our current unfortunate observational sensitivity to a distance range of tenths of au (where we do not see any moons in the Solar system).

The data we have on exo-planets is typically not as accurate as those for the Solar system planets, and contains missing parameters. For example, exo-planets detected by transit photometry but not confirmed with Doppler radial velocity measurements do not have measured masses. Exoplanets detected by radial velocity measurements but not with transit have no radius measurements, and only lower bounds on mass. Transiting exo-planets sometimes do not have secure eccentricity values, meaning that their apocentric and pericentric distances are uncertain. Further, other physical effects which could have significant impacts on transits (but not treated here) such as oblateness, tidal distortions, and extended atmospheres are often or always not well-constrained in exoplanetary systems.

Despite these caveats, we can analyze some systems of interest: TRAPPIST-1 (Gillon et al. 2017), Kepler-444 (Campante et al. 2015) and Kepler-47 (Orosz et al. 2012). TRAPPIST-1 and Kepler-444 are single-star 7-planet and 5-planet systems, containing all terrestrial/rocky planets of radii between about $0.4-1.2 R_{\oplus}$. Kepler- 47 is a 2 -planet circumbinary planetary system with two planets of radii $3.0 R_{\oplus}$ and $4.7 R_{\oplus}$. Conveniently, all planets in all systems are lettered in alphabetical order according to their distance from the parent star(s).

By using semimajor axis values to represent distances, we compute some quantities of interest. Figure 4 displays the maximum eclipse depth $(g)$ versus the maximum angular diameter $\left(\phi_{23}\right)$ for three-body combinations in these systems. The two highest eclipse depths both arise from the circumbinary Kepler-47 system, and viewers on the outermost planet (d) in the TRAPPIST-1 system will see very similar transit features from each of the inner planets (b and $c$ ). In that system, the transit of planet $\mathrm{b}$ as viewed from planet $\mathrm{c}$ has the largest angular diameter: over one degree.

We take a closer look at the TRAPPIST-1 system in Table 2, where we compute eclipse quantities for the three targets which are most likely to be habitable (planets e, f and $\mathrm{g}$ ). In no case on these planets will a total eclipse occur, and in all cases the eclipse depth is between one and ten per cent and the maximum angular diameter between about ten and thirty arcseconds.

\section{APPLICATION TO ANTICIPATED EXO-SYSTEMS}

The discovery of exo-moons will inevitably introduce questions about whether and what types of eclipses will occur. The formalism here can be applied directly to such situations. As a proof-of-concept, imagine that a large moon was found orbiting a Jupiter in a low-mass star system, such that $R_{1}=0.2 R_{\odot}, R_{2}=R_{\text {Jup }}$ and $R_{3}=0.5 R_{\oplus}$, with $r_{12}=1 \mathrm{au}$ and $r_{23}=385 \times 10^{3} \mathrm{~km}$ (the mean Moon-Earth distance). In this case, the moon would experience total eclipses, and, as viewed from the moon, $\max \left(\phi_{23}\right) \approx 6.4^{\prime}$.

Another type of planetary system include planets orbiting stars at the point during their evolution - the asymptotic giant branch phase - when the host star radius is maximum. So far, only one planet candidate has been reported to orbit such a star (Kervella et al. 2016), but only after the stellar radius has contracted following a period of expansion. Suppose instead that the star had $R_{1}=1.5$ au and hosted two Earth-like planets $\left(R_{2}=R_{\oplus}\right.$ and $\left.R_{3}=R_{\oplus}\right)$. Suppose these planets were sufficiently far $\left(r_{12}=2.5 \mathrm{au}\right.$ and $\left.r_{23}=3.0 \mathrm{au}\right)$ to have survived the tidal pull of the star on the asymptotic giant branch (Mustill \& Villaver 2012). Then an annu- 


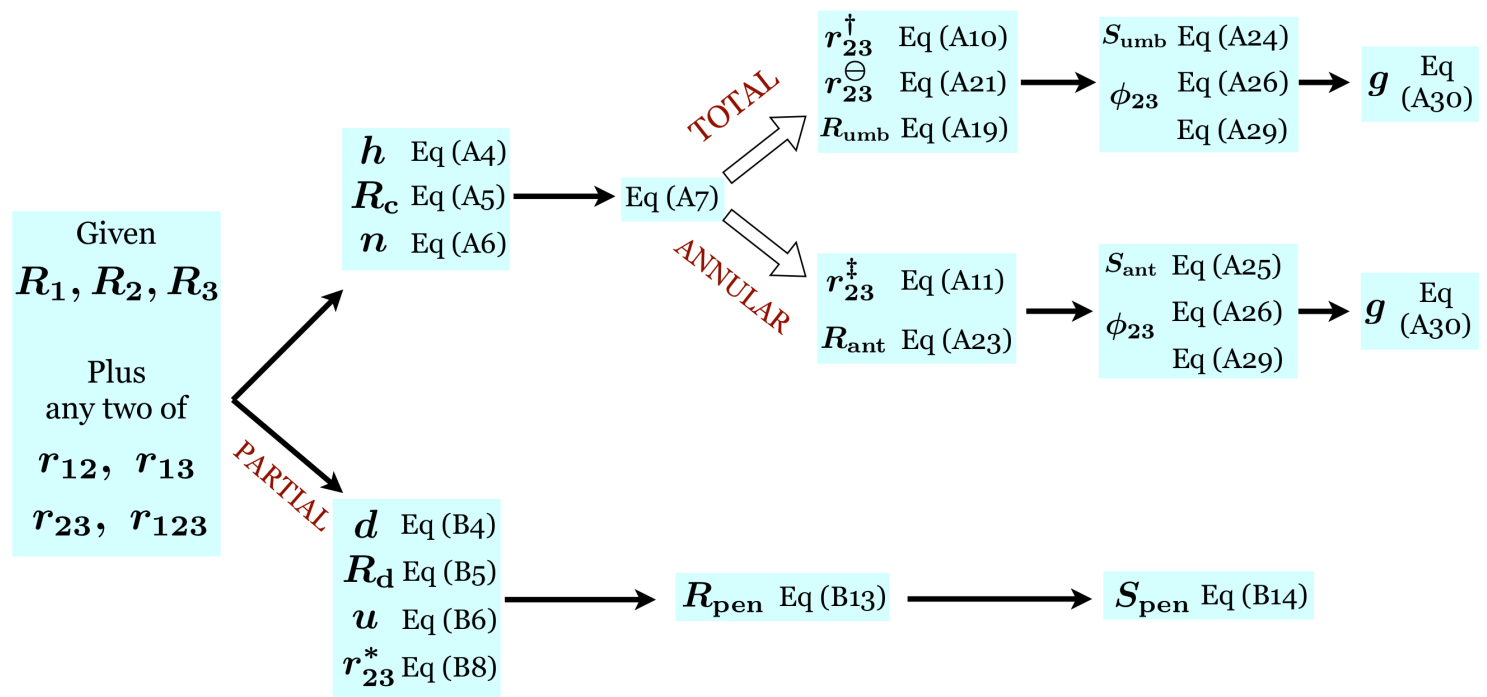

Figure 5. Algorithm to compute eclipse properties given only radii and mutual distances.

lar eclipse would occur, and despite the relatively enormous stellar size, $\max \left(\phi_{23}\right)=0.59^{\prime}$. However, $g=2.6 \times 10^{-8}$, producing a negligible change in brightness of the giant star.

Now consider white dwarf planetary systems. At least 5 of the Solar system planets will survive into the Solar white dwarf phase (Veras 2016a b) , and signatures of planetary debris persist in the atmospheres of over 30 per cent of all white dwarfs (Koester et al. 2014) and is detected in circumstellar discs (Farihi 2016). Planets are hence expected to orbit white dwarfs at distances of a few au. A white dwarf hosting two Earth-like planets then feature syzygys where all three objects are nearly the same size. Imagine that $R_{1}=R_{\oplus}$, $R_{2}=0.95 R_{\oplus}, R_{3}=0.95 R_{\oplus}, r_{12}=2.5$ au and $r_{13}=3.0$ au. Then a total eclipse will occur such that even though the umbral shadow does not quite engulf the target $\left(R_{\text {umb }}=5990\right.$ $\mathrm{km}$ ), the penumbral shadow does. Despite the total eclipse, the maximum angular diameter which would be eclipsed is just 0.97 '

\section{SUMMARY}

Syzygys are common features of planetary systems, including our own. Here, we have constructed an infundibuliform geometry which characterises eclipses, transits and occultations under one framework. Given only radii and mutual distances, several quantities - such as eclipse type, shadow radii and transit depth - can be computed with basic trigonometry (Appendices A and B). We illustrate a user-friendly equation flow detailing these computations in Fig. 5.

We have also analyzed three classes of special cases, when the occulter is a planet (Section 3), star (Section 4) and moon (Section 5). We found that total eclipses cannot occur in two-planet systems with a main sequence or giant branch parent star, but may occur often for two-planet systems containing a white dwarf. In circumbinary systems with circular orbits and similarly dense stars, by appealing to stability criteria we have parametrized the condition for total eclipses to never occur to just two variables: the stellar radii (equation 13). The relations in this paper represent useful estimates which may be employed in mission planning and for added perspective on a wide variety of exo-systems.

\section{ACKNOWLEDGEMENTS}

We thank the referee for helpful comments on the manuscript. DV has received funding from the European Research Council under the European Union's Seventh Framework Programme (FP/2007-2013)/ERC Grant Agreement n. 320964 (WDTracer).

\section{REFERENCES}

Anglada-Escudé, G., Amado, P. J., Barnes, J., et al. 2016, Nature, 536, 437

Armstrong, D. J., Osborn, H. P., Brown, D. J. A., et al. 2014, MNRAS, 444, 1873

Batygin, K., \& Brown, M. E. 2016, AJ, 151, 22

Braga-Ribas, F., Sicardy, B., Ortiz, J. L., et al. 2014, Nature, 508, 72

Campante, T. L., Barclay, T., Swift, J. J., et al. 2015, ApJ, 799,170

de la Fuente Marcos, C., \& de la Fuente Marcos, R. 2014, MNRAS, 443, L59

Domingos, R. C., Winter, O. C., \& Yokoyama, T. 2006, MNRAS, 373, 1227

Donnison, J. R. 2010, MNRAS, 406, 1918

Farihi, J. 2016, New Astronomy Reviews, 71, 9

Gillon, M. et al. 2017, Nature, 542, 456

Hamilton, D. P., \& Krivov, A. V. 1997, Icarus, 128, 241

Heller, R. 2017, arXiv:1701.04706

Holman, M. J., \& Wiegert, P. A. 1999, AJ, 117, 621

Iorio, L. 2014, MNRAS, 444, L78

Kenworthy, M. A., \& Mamajek, E. E. 2015, ApJ, 800, 126

Kervella, P., Homan, W., Richards, A. M. S., et al. 2016, A\&A, 596, A92

Koester, D., Gänsicke, B. T., \& Farihi, J. 2014, A\&A, 566, A34 
Konacki, M., \& Wolszczan, A. 2003, ApJL, 591, L147

Kostov, V. B., Orosz, J. A., Welsh, W. F., et al. 2016, ApJ, 827,86

Li, Y., \& Zhang, C. Z. 1998, A\&A, 332, 1142

Martin, D. V., \& Triaud, A. H. M. J. 2015, MNRAS, 449, 781

McDonald, A. J. C. 1986, Celestial Mechanics, 38, 139

Mustill, A. J., \& Villaver, E. 2012, ApJ, 761, 121

Orosz, J. A., Welsh, W. F., Carter, J. A., et al. 2012, Science, 337, 1511

Payne, M. J., Veras, D., Holman, M. J., Gänsicke, B. T. 2016a, MNRAS, 457, 217

Pease, G. E., \& Glenn, G. S. 2016, arXiv:1610.03553

Ragozzine, D., \& Holman, M. J. 2010, arXiv:1006.3727

Sahlmann, J., Triaud, A. H. M. J., \& Martin, D. V. 2015, MNRAS, 447, 287

Veras, D. 2016a, Royal Society Open Science, 3, 150571

Veras, D. 2016b, MNRAS, 463, 2958

Veras, D., Carter, P. J., Leinhardt, Z. M., \& Gänsicke, B. T. 2017, MNRAS, 465, 1008

Wolszczan, A. 1994, Science, 264, 538

In Appendices $\mathrm{A}$ and $\mathrm{B}$, we present the geometries which form the basis of the results of this paper, and derive key equations used in the main body of the text.

\section{APPENDIX A: TOTAL AND ANNULAR ECLIPSES}

Figure 1 displays a schematic for the cone formed from external tangent lines. If the target at some point along its orbit intersects this cone, then the target is in the umbra and a total eclipse will occur. Otherwise, the target will be in the antumbra and an annular eclipse will occur.

Our first goal is to find the height, $h$, of the cone in terms of the given variables (the radii $R$ of all three objects and their centre-to-centre distances $r$ ). To do so, first consider Fig. A1. The location of the base of the cone is offset from the centre of the primary by a distance $n$, and the radius of the cone base is $R_{\mathrm{c}}$. Trigonometry gives

$$
\begin{aligned}
n & =\frac{R_{\mathrm{c}}^{2}}{h} \\
h+n & =\frac{R_{1} r_{12}}{R_{1}-R_{2}}, \\
R_{\mathrm{c}}^{2} & =\frac{1}{2}\left[h \sqrt{h^{2}+4 R_{1}^{2}}-h^{2}\right]
\end{aligned}
$$

such that their simultaneous solution yields a cone height of

$h=R_{1}\left[\frac{r_{12}}{R_{1}-R_{2}}-\frac{R_{1}-R_{2}}{r_{12}}\right]$.

Consequently, the base of the cone is

$R_{\mathrm{c}}=\left(\frac{R_{1}}{r_{12}}\right) \sqrt{r_{12}^{2}-\left(R_{1}-R_{2}\right)^{2}}$

and

$n=\frac{R_{1}}{r_{12}}\left(R_{1}-R_{2}\right)$.

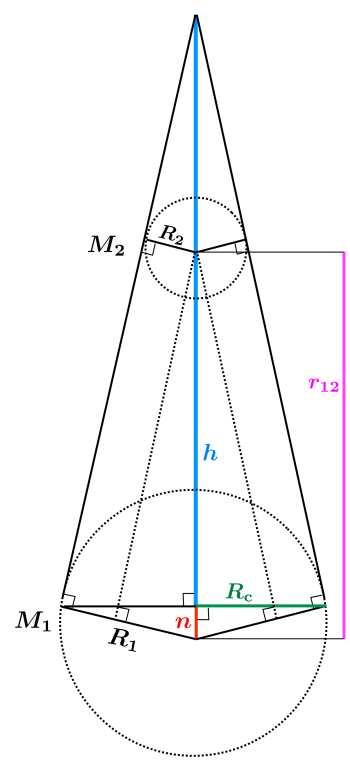

Figure A1. Zoom-in of the umbral cone from Fig. 1 The radius of the base of the cone is $R_{\mathrm{C}}$, and its height is $h$. Equations A1 A3) were derived based on the geometry in this diagram.

Note that in equations (A4 $\mathrm{A} 6$, as $R_{2} \rightarrow R_{1}$, we obtain the expected results $n \rightarrow 0, R_{\mathrm{c}} \rightarrow R_{1}$ and $h \rightarrow \infty$.

\section{A1 Eclipse criteria}

Either a total or annular eclipse will occur at syzygy depending on the location of the target with respect to the umbral cone. If the target is beyond the tip of the cone, then the eclipse is annular. Consequently, the condition may be expressed in variables as

$h<r_{13}-n-R_{3}$, annular eclipse

$h \geqslant r_{13}-n-R_{3}$, total eclipse

\section{A2 Shadow radii}

\section{A2.1 Total coverage}

In order to determine if the umbral or antumbral shadows will envelope the target completely, consider Fig. A2 which presents limiting cases for a static occulter given that the value of $r_{12}$ is fixed. For the umbral case, let $r_{23}^{\dagger}$ represent the critical value of $r_{23}$ within which $\left(r_{23} \leqslant r_{23}^{\dagger}\right)$ the target is engulfed in the total eclipse. For the antumbral case, denote $r_{23}^{\ddagger}$ as the value beyond which $\left(r_{23} \geqslant r_{23}^{\ddagger}\right)$ the entire target is engulfed in the annular eclipse. By entirely engulfed, we are referring to when the entire target is within the umbral or antumbral cone.

Similar triangles from the left-hand panel give

$\frac{R_{1}}{h+n}=\frac{R_{2}}{h+n-r_{12}}=\frac{R_{3}}{h+n-r_{12}-r_{23}^{\dagger}}$

and

$\frac{R_{1}}{h+n}=\frac{R_{2}}{h+n-r_{12}}=\frac{R_{3}}{-\left(h+n-r_{12}-r_{23}^{\ddagger}\right)}$, 

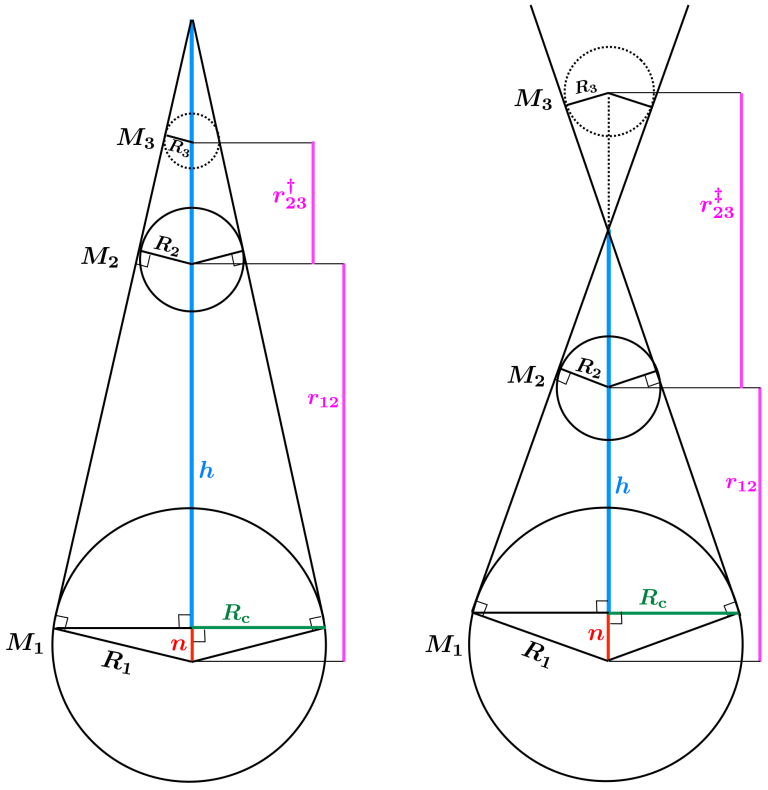

Figure A2. The limiting target-occulter distances $\left(r_{23}^{\dagger}\right.$ and $\left.r_{23}^{\ddagger}\right)$ for which the entire target is covered in the umbra (left-hand panel) or antumbra (right-hand panel). For $r_{23} \leqslant r_{23}^{\dagger}$, the target is entirely engulfed in the umbra. For $r_{23} \geqslant r_{23}^{\ddagger}$, the target is entirely engulfed in the antumbra. Equations (A81313 were derived from the geometry in this figure.

yielding

$r_{23}^{\dagger}=r_{12}\left(\frac{R_{2}-R_{3}}{R_{1}-R_{2}}\right)$,

$r_{23}^{\ddagger}=r_{12}\left(\frac{R_{2}+R_{3}}{R_{1}-R_{2}}\right)$.

Equation A10 demonstrates that in order for a total eclipse to engulf the entire target, it must be smaller than the occulter. Alternatively, Equation (A11) illustrates that any target can be entirely engulfed by the antumbral shadow if sufficiently far from the occulter.

These equations may be turned around to solve for any of the other variables given all others. For example, for a given value of $r_{23}$, the critical target radii for umbral and antumbral engulfment are

$R_{3}^{\dagger}=R_{2}-\frac{r_{23}}{r_{12}}\left(R_{1}-R_{2}\right)$,

$R_{3}^{\ddagger}=\frac{r_{23}}{r_{12}}\left(R_{1}-R_{2}\right)-R_{2}$.

At $r_{23}^{\dagger}$ or $R_{3}^{\dagger}$, more than half of the surface of the target will be exposed to the umbral shadow. The projected shadow radius $R_{\text {umb }}$ (see Fig. 1) in this specific case (Fig. A2) is given by

$\cos \left(\sin ^{-1}\left[\frac{R_{\mathrm{umb}}^{\dagger}}{R_{3}}\right]\right)=\frac{R_{1}}{h+n}$

or

$R_{\mathrm{umb}}^{\dagger}=\left(\frac{R_{3}}{r_{12}}\right) \sqrt{r_{12}^{2}-\left(R_{1}-R_{2}\right)^{2}}=\frac{R_{\mathrm{c}} R_{3}}{R_{1}}$

In contrast, at $r_{23}^{\ddagger}$ or $R_{3}^{\ddagger}$, less than half of the surface of the target will be exposed to the antumbral shadow. However, geometry illustrates that the projected radius of the shadow $R_{\text {ant }}$ takes the same form as $R_{\mathrm{umb}}$ and is

$R_{\text {ant }}^{\ddagger}=R_{\text {umb }}^{\dagger}$.

\section{A2.2 Some umbral coverage}

Now we derive more general formulae for $R_{\mathrm{umb}}$ and $R_{\mathrm{ant}}$ which are not bound to the geometry in Fig. A2 We begin with the umbral case. Consider when $h \geqslant r_{13}-n-R_{3}$ and $r_{23}>r_{23}^{\dagger}$, such that a total eclipse occurs and the target is not completely engulfed in the umbra, as in Fig. 1. If the base of the cone is flush with the $x-y$ Cartesian plane, then the equation of a double cone (extending in opposite directions) would be

$\left(\frac{h}{R_{\mathrm{c}}}\right)^{2}\left(x^{2}+y^{2}\right)=(z-h)^{2}$

so that if a total eclipse occurred on the target, the radius of the projected area of totality, $R_{\mathrm{umb}}=\sqrt{x^{2}+y^{2}}$, would be

$R_{\mathrm{umb}}=R_{\mathrm{c}}\left(1-\frac{r_{13}-n-\sqrt{R_{3}^{2}-R_{\mathrm{umb}}^{2}}}{h}\right)$.

Solving for $R_{\text {umb }}$ gives

$R_{\mathrm{umb}}=\left(\frac{h R_{\mathrm{c}}}{h^{2}+R_{\mathrm{c}}^{2}}\right)\left[h+n-r_{13}\right.$

$$
\left.+\sqrt{R_{3}^{2}-\left(\frac{R_{\mathrm{c}}}{h}\right)^{2}\left[\left(h+n-r_{13}\right)^{2}-R_{3}^{2}\right]}\right]
$$

which is consistent with the condition given by equation (A7).

Also, recall that when the target is completely engulfed in the umbral shadow, then over half of the surface is exposed to this shadow, such that $R_{3}>R_{\mathrm{umb}}^{\dagger}$. What if the target is just large enough so that $R_{3}=R_{\mathrm{umb}}^{\dagger}$ ? Define $R_{\mathrm{umb}}^{\ominus}$ as the value of $R_{3}$ such that $R_{3}=R_{\mathrm{umb}}^{\dagger}$, and $r_{23}^{\ominus}$ as the value of $r_{23}$ such that $R_{3}=R_{\mathrm{umb}}^{\dagger}$. Then equation A18 reduces to

$R_{\mathrm{umb}}^{\ominus}=\left(\frac{R_{\mathrm{c}}}{h}\right)\left(h+n-r_{13}\right)$

or, alternatively,

$r_{23}^{\ominus}=\frac{h}{R_{\mathrm{c}}}\left(R_{\mathrm{c}}-R_{3}\right)+n-r_{12}$.

The value of $r_{23}^{\ominus}$ represents a useful quantity to access when computing the surface area covered by the shadow.

\section{A2.3 Some antumbral coverage}

Now we find a general formula for $R_{\text {ant }}$. If an annular eclipse occurs and $r_{23}<r_{23}^{\ddagger}$, then the equation of the appropriate cone in this instance yields

$R_{\mathrm{ant}}=R_{\mathrm{c}}\left(\frac{r_{13}-n-\sqrt{R_{3}^{2}-R_{\mathrm{ant}}^{2}}}{h}-1\right)$

or

(C) 0000 RAS, MNRAS 000, 1 13 


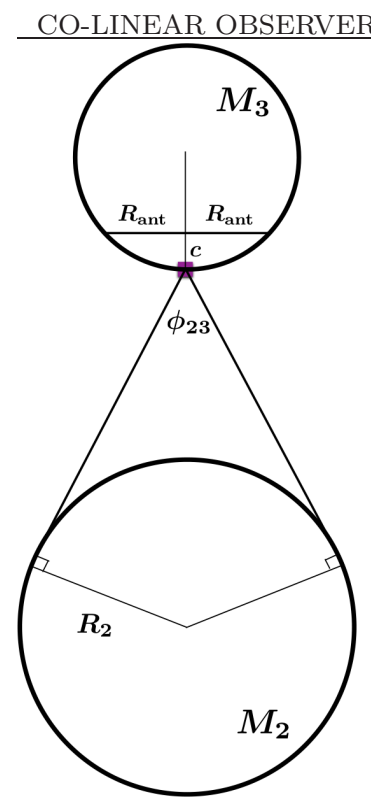

OFFSET OBSERVER

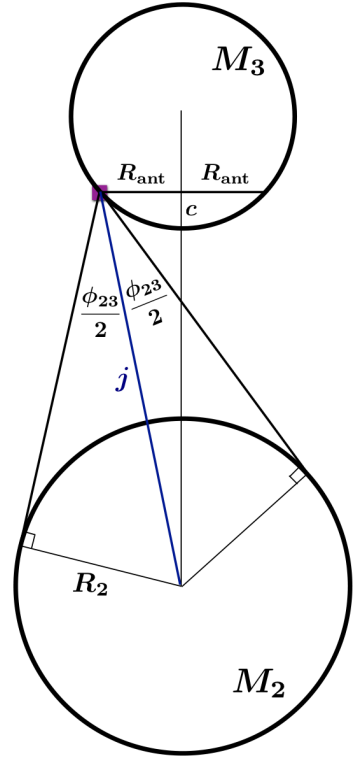

Figure A3. Angular diameter $\left(\phi_{23}\right)$ of occulter when the viewer (standing on the target $M_{3}$ ) is co-linear with the syzygy (lefthand panel) and when the viewer (square) is at the edge of the umbral or antumbral shadow (right-hand panel). These situations respectively maximize and minimize the value of $\phi_{23}$. Although $R_{\text {ant }}$ is used in the diagrams, equally $R_{\text {umb }}$ may be adopted. Equations A26, A28 were derived from the geometry here.

$$
\begin{aligned}
R_{\mathrm{ant}}= & \left(\frac{h R_{\mathrm{c}}}{h^{2}+R_{\mathrm{c}}^{2}}\right)\left[r_{13}-h-n\right. \\
& \left.-\sqrt{R_{3}^{2}-\left(\frac{R_{\mathrm{c}}}{h}\right)^{2}\left[\left(r_{13}-h-n\right)^{2}-R_{3}^{2}\right]}\right]
\end{aligned}
$$

which is also consistent with the condition given by equation (A7).

\section{A3 Shadow surface areas}

Due to geometry of spherical caps, the surface area of the target which is eclipsed at syzygy in the umbra, $S_{\text {umb }}$ is

$$
\begin{aligned}
S_{\mathrm{umb}}=\pi & {\left[R_{\mathrm{umb}}^{2}+\left(R_{3}-\sqrt{R_{3}^{2}-R_{\mathrm{umb}}^{2}}\right)^{2}\right], } \\
& r_{23} \geqslant r_{23}^{\ominus} ; \\
= & 4 \pi R_{3}^{2}-\pi\left[R_{\mathrm{umb}}^{2}+\left(R_{3}-\sqrt{R_{3}^{2}-R_{\mathrm{umb}}^{2}}\right)^{2}\right], \\
& r_{23}^{\dagger} \leqslant r_{23}<r_{23}^{\ominus} .
\end{aligned}
$$

For $r_{23}<r_{23}^{\dagger}$, the entire target is engulfed and is no longer defined. In the antumbra,

$S_{\mathrm{ant}}=\pi\left[R_{\mathrm{ant}}^{2}+\left(R_{3}-\sqrt{R_{3}^{2}-R_{\mathrm{ant}}^{2}}\right)^{2}\right], \quad r_{23} \leqslant r_{23}^{\ddagger}($

such that for $r_{23}>r_{23}^{\ddagger}, R_{\text {ant }}$ is no longer defined.

\section{A4 Angular diameters}

During syzygy, an observer standing on the target in the umbral and antumbral shadow will see some or none of the radiating primary. Let $\phi_{13}$ represent the angular diameter of the primary supposing that the occulter does not exist, and let $\phi_{23}$ represent the angular diameter of the occulter. In diagrams throughout the paper, we represent the observer as a square.

\section{A4.1 Co-linear observers}

For both total and annular eclipses, during syzygy, an observer standing on the target anywhere within the shadow will see the primary at least partly obscured by the occulter. The maximum value of $\phi_{23}$ is achieved when the observer is co-linear with the syzygy (left panel of Fig. A3). Then, regardless of the relative size of the target and occulter,

$$
\begin{aligned}
\max \left(\phi_{23}\right) & =2 \sin ^{-1}\left(\frac{R_{2}}{r_{23}-R_{3}}\right), & & h<r_{13}-n-R_{3} \\
& =2 \sin ^{-1}\left(\frac{R_{1}}{r_{13}-R_{3}}\right), & & h \geqslant r_{13}-n-R_{3}(\mathrm{~A} 26)
\end{aligned}
$$

such that the upper branch corresponds to an annular eclipse, and the lower branch a total eclipse. The expression in the lower branch is equal to $\max \left(\phi_{13}\right)$ for both annular and total eclipses.

\section{A4.2 Offset observers}

When the observer is not co-linear with the syzygy, but remains in the umbra or antumbra, then the angular diameters are smaller. Regardless of the location of the observer within the shadow, they will see the entire occulter creating an obscuration on the primary's disc. For a given architecture, the minimum possible value of $\phi_{23}$ is then achieved when the observer is at the edge of the shadow (right panel of Fig. A3). Using the helpful auxiliary variables

$$
\begin{aligned}
& c=R_{3}-\sqrt{R_{3}^{2}-R_{\mathrm{ant}}^{2}}, \\
& j=\sqrt{\left(r_{23}-R_{3}+c\right)^{2}+R_{\mathrm{ant}}^{2}} .
\end{aligned}
$$

gives

$$
\begin{aligned}
& \min \left(\phi_{23}\right)= \sin ^{-1}\left[\frac{R_{2}}{\sqrt{\left(r_{23}-\sqrt{R_{3}^{2}-R_{\mathrm{ant}}^{2}}\right)^{2}+R_{\mathrm{ant}}^{2}}}\right], \\
& 2 \sin ^{-1}\left[\frac{R_{1}}{h<r_{13}-n-R_{3} ;}\right. \\
& {\left[\begin{array}{l}
\sqrt{\left(r_{13}-\sqrt{R_{3}^{2}-R_{\mathrm{umb}}^{2}}\right)^{2}+R_{\mathrm{umb}}^{2}} \\
h \geqslant r_{13}-n-R_{3}
\end{array},\right.}
\end{aligned}
$$



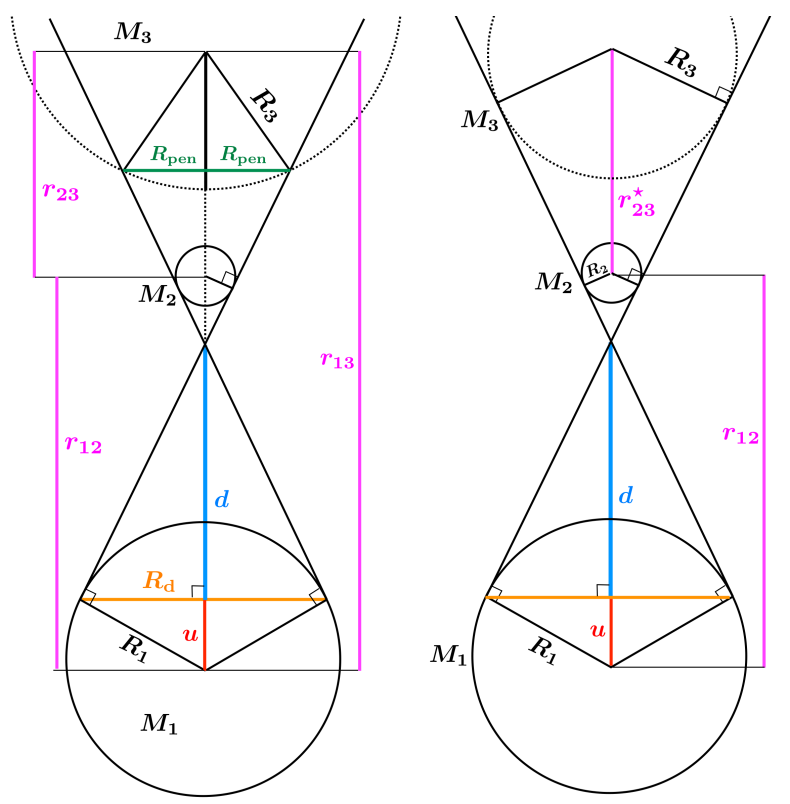

Figure A4. A more detailed geometrical diagram of Fig. 2 (left panel) and the limiting target-occulter distance $\left(r_{23}^{*}\right)$ for which the entire target is covered in the penumbra (right-hand panel). For $r_{23} \geqslant r_{23}^{*}$, the target is engulfed in the penumbral shadow. Equations B1.B9 were derived based on the geometry in this diagram.

such that the upper branch corresponds to an annular eclipse, and the lower branch a total eclipse. Again, the expression in the lower branch is equal to $\min \left(\phi_{13}\right)$ for both annular and total eclipses. These results hold regardless of whether the target or occulter is larger.

\section{A4.3 Useful benchmark}

The prominence of the eclipse can be measured by the fractional area of the primary blocked out by the occulter

$g \equiv\left(\frac{\phi_{23}}{\phi_{13}}\right)^{2}$

such that a total eclipse corresponds to $g=1$. Here, $g$ would be equivalent to the so-called "transit depth", which is a useful measure in studies of some exo-planetary systems.

\section{APPENDIX B: PARTIAL ECLIPSES}

If the target is sufficiently large, then regardless if total or annular eclipses occur at syzygy, partial eclipses will occur concurrently for some observers who are outside of the umbral or antumbral shadow The penumbral shadow is generated from the cone containing the occulter that is drawn from internal tangent lines: Figure 2 displays a schematic. We proceed along similar lines in this section as with the last.

Consider the geometry in the left panel of Fig. A4 The height of the penumbral double cone is $d$, and the radius of its base is $R_{\mathrm{d}}$. One obtains

$$
u=\frac{R_{\mathrm{d}}^{2}}{d}
$$

$$
\begin{aligned}
d+u & =\frac{R_{1} r_{12}}{R_{1}+R_{2}}, \\
R_{\mathrm{d}}^{2} & =\frac{1}{2}\left[d \sqrt{d^{2}+4 R_{1}^{2}}-d^{2}\right],
\end{aligned}
$$

Comparison with equations A1 A3 reveals that the only difference is in the denominator of equation A1. The simultaneous solution gives

$$
\begin{aligned}
& d=R_{1}\left[\frac{r_{12}}{R_{1}+R_{2}}-\frac{R_{1}+R_{2}}{r_{12}}\right], \\
& R_{\mathrm{d}}=\left(\frac{R_{1}}{r_{12}}\right) \sqrt{r_{12}^{2}-\left(R_{1}+R_{2}\right)^{2}}, \\
& u=\frac{R_{1}}{r_{12}}\left(R_{1}+R_{2}\right) .
\end{aligned}
$$

Therefore, $d, R_{\mathrm{d}}$ and $u$ can be obtained from $h, R_{\mathrm{c}}$ and $n$ (equations A1 A3 just by substituting $R_{2}$ with $\left(-R_{2}\right)$.

\section{B1 Shadow radii}

First, we determine the limiting conditions under which the entire target is engulfed in the penumbra.

\section{B1.1 Total coverage}

This task is aided by the right panel of Fig. A4 which assumes a static occulter given a fixed value of $r_{12}$. Let $r_{23}^{*}$ represent the critical value of $r_{23}$ beyond which the target is engulfed in the penumbral shadow. Then

$\frac{R_{1}}{d+u}=\frac{R_{2}}{r_{12}-d-u}=\frac{R_{3}}{r_{23}^{*}+r_{12}-d-u}$

and

$r_{23}^{*}=r_{12}\left(\frac{R_{3}-R_{2}}{R_{1}+R_{2}}\right)$.

Equation (B8) may be compared to equations (A10)-A11, and shows that if the target is smaller than the occulter, then the target will always be engulfed. We can also write

$R_{3}^{*}=R_{2}+\left(R_{1}+R_{2}\right) \frac{r_{23}}{r_{12}}$

At $r_{23}^{*}$ or $R_{3}^{*}$, less than half of the target will be bathed in the partial eclipse, similarly to an antumbral eclipse. The projected radius of the shadow $R_{\text {pen }}$ (see Fig. (2) in this specific case (right-hand panel of Fig. A4) is given by

$\cos \left(\sin ^{-1}\left[\frac{R_{\text {pen }}^{*}}{R_{3}}\right]\right)=\frac{R_{1}}{d+u}$

or

$R_{\text {pen }}^{*}=\left(\frac{R_{3}}{r_{12}}\right) \sqrt{r_{12}^{2}-\left(R_{1}+R_{2}\right)^{2}}=\frac{R_{\mathrm{d}} R_{3}}{R_{1}}$.

\section{B1.2 Some coverage}

When the target is not completely engulfed in the penumbra $\left(r_{23}^{*}>r_{23}\right)$, then in order to determine the shadow radius $R_{\text {pen }}$, we again appeal to the properties of double cones. See the left panel of Fig. A4 We can make the following substitutions in equation A17: $h \rightarrow d, R_{\mathrm{c}} \rightarrow R_{\mathrm{d}},\left(x^{2}+y^{2}\right) \rightarrow R_{\text {pen }}^{2}$ and $z \rightarrow r_{13}-u-\sqrt{R_{3}^{2}-R_{\text {pen }}^{2}}$. Then, by analogy with the antumbral case, 


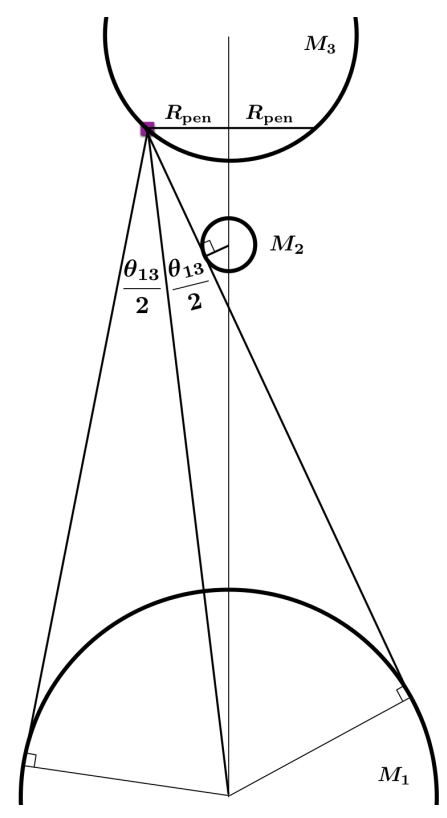

Figure B1. Angular diameter $\left(\theta_{13}\right)$ of primary when the viewer (square) is standing at the edge of the penumbral shadow on the target $M_{3}$. In this instance, the occulter does not block the primary at all, giving $\theta_{23}=0$.

$R_{\mathrm{pen}}=R_{\mathrm{d}}\left(\frac{r_{13}-u-\sqrt{R_{3}^{2}-R_{\mathrm{pen}}^{2}}}{d}-1\right)$

or

$$
\begin{aligned}
R_{\mathrm{pen}}= & \left(\frac{d R_{\mathrm{d}}}{d^{2}+R_{\mathrm{d}}^{2}}\right)\left[r_{13}-d-u\right. \\
& \left.-\sqrt{R_{3}^{2}-\left(\frac{R_{\mathrm{d}}}{d}\right)^{2}\left[\left(r_{13}-d-u\right)^{2}-R_{3}^{2}\right]}\right]
\end{aligned}
$$

\section{B2 Surface area}

The surface area of the target which experiences the partial eclipse is well-defined as either $\left(S_{\text {pen }}-S_{\text {ant }}\right)$ or $\left(S_{\text {pen }}-S_{\text {umb }}\right)$ provided that $R_{\text {ant }}$ or $R_{\text {umb }}$ exist and where $S_{\mathrm{pen}}=\pi\left[R_{\mathrm{pen}}^{2}+\left(R_{3}-\sqrt{R_{3}^{2}-R_{\mathrm{pen}}^{2}}\right)^{2}\right], \quad r_{23} \leqslant r_{23}^{*}$

We assume that $R_{\text {pen }}$ does not exist if $r_{23}>r_{23}^{*}$.

\section{B3 Angular diameters}

\section{B3.1 Co-linear observers}

At syzygy, co-linear observers never experience a partial eclipse. They are instead in the umbra or antumbra, and so will experience a total eclipse.

\section{B3.2 Offset observers at maximum coverage}

Denote the angular diameters of the eclipsed occulter and primary as seen from an observer (on the target) in the penumbra as $\theta_{23}$ and $\theta_{12}$. The maximum value of $\theta$ occurs when an observer resides at the boundary between the penumbral shadows and the umbral or antumbral shadows. Hence,

$\max \left(\theta_{23}\right)=\min \left(\phi_{23}\right)$,

$\max \left(\theta_{13}\right)=\min \left(\phi_{13}\right)$.

\section{B3.3 Offset observers at minimum coverage}

The minimum values of $\theta$ are achieved at the edge of the shadow. Geometry dictates that at that location, the target and occulter appear in their entirety and are grazing. Figure B1 illustrates that

$\min \left(\theta_{23}\right)=0$

whereas

$\min \left(\theta_{13}\right)=2 \sin ^{-1}\left[\frac{R_{1}}{\sqrt{\left(r_{13}-\sqrt{R_{3}^{2}-R_{\text {pen }}^{2}}\right)^{2}+R_{\text {pen }}^{2}}}\right]$ 